\title{
Civilisations
}

Revue internationale d'anthropologie et de sciences

humaines

$48 \mid 2001$

La question de l'islam et de l'Etat à l'aube du XXle siècle

\section{Est-ce que les musulmans peuvent être anglais ?}

Reformuler les identités ethniques dans le Royaume-Uni

\section{John Eade}

Traducteur : Sian JONES

\section{CpenEdition}

\section{Journals}

Édition électronique

URL : http://journals.openedition.org/civilisations/3476

DOI : $10.4000 /$ civilisations.3476

ISSN : 2032-0442

\section{Éditeur}

Institut de sociologie de l'Université Libre de Bruxelles

\section{Édition imprimée}

Date de publication : 31 août 2001

Pagination : 161-175

ISSN : 0009-8140

\section{Référence électronique}

John Eade, «Est-ce que les musulmans peuvent être anglais ? ", Civilisations [En ligne], 48 | 2001, mis en ligne le 01 août 2004, consulté le 07 mai 2019. URL : http://journals.openedition.org/ civilisations/3476 ; DOI : 10.4000/civilisations.3476 


\section{LE DÉBAT SUR L'ISLAM ET LA DÉMOCRATIE OU QUAND L'IMAGINAIRE L'EMPORTE}

\section{Gema MARTÍN-MUÑoZ}

«Islam has not been hospitable to democracy», l'islam n'a guère été accueillant vis à vis de la démocratie, disait Samuel Huntington en 1984. Ce qui veut dire qu'en matière de démocratie, l'islam n'a pas de sources où puiser. Cette affirmation de Huntington se faisait l'écho d'une tendance, malheureusement courante, non seulement dans les médias, mais même chez les spécialistes du monde arabe. Mais si l'on se donne la peine d'étudier attentivement les analyses qui suivent cette hypothèse, on en arrive à la conclusion qu'elles sont avant tout le fruit d'une attitude volontariste, de méthodologies déterministes et «essentialistes» où le «spécifique» (ce qui relève de «l'essence») sert à tout expliquer. Ces théories expliquent le déficit démocratique des pays musulmans par de soi-disant «défauts congénitaux» de l'Islam et des Arabes. C'est à dire par des facteurs inhérents, immuables et déterminants de la culture arabe ou musulmane qui prévaut dans la région, et non par des expériences historiques et des facteurs sociopolitiques et stratégiques qui peuvent changer. On trouve de nombreuses théories fondées sur l'hypothèse d'une incapacité à mettre en œuvre la démocratie dans une culture politique qui serait marquée par le «sultanisme», ou encore focalisée sur l'utopie de l'État islamique (Pipes, 1983). Cette incapacité s'expliquerait également par la «culture islamique», où il ne saurait y avoir d'espace autonome pour la politique, et où espace public et privé se confondent (Badie, 1986). Ou encore parce qu'on considère que le principe de liberté ne peut pas s'enraciner dans ces sociétés étant donné que c'est le principe de justice qui a prévalu dans la tradition islamique, et que la communauté l'emportera toujours sur l'individu (Lewis, 1996).

De plus, il semble même que les spécialistes s'efforcent toujours de laisser les musulmans en marge des courants de pensée du moment. Ainsi lorsqu'un consensus règne en sociologie pour considérer que la démocratie et ie développement impliquent nécessairement l'action de groupes sociaux forts et dynamiques, on voit tout un courant important d'orientalistes décréter que de tels mouvements associatifs n'existent pas en Islam. Le caractère totalitaire de la foi (il est d'ailleurs difficile de comprendre pourquoi celui-ci est attribué exclusivement à l'islam alors qu'il est commun à toutes les religions monothéistes) impliquerait que seul un État luimême totalitaire soit en mesure d'appliquer le dogme de la religion et que l'islam décourage la formation de groupes capables de s'opposer au despotisme. 
La conclusion à laquelle on arrive avec ce genre de théories c'est que, dans les sociétés musulmanes, l'État est plus fort que la société. Faiblement organisées et dépourvues d'identité corporative forte, les associations sociopolitiques du monde musulman seraient l'exception, et les groupes informels la règle; ceux-ci serviraient d'ailleurs avant tout d'instrument de collaboration et de soutien au pouvoir et à ses élites, dont les liens politiques relèvent du clientélisme (Bill \& Springborg, 1984).De même, lorsque la sociologie politique défend l'idée qu'une société civile forte est indispensable à la mise en place du processus de démocratisation, les mêmes arguments sont alors utilisés pour démontrer que les pays musulmans sont incapables de répondre à une telle exigence (Sadowsky, $1993: 19$ ).

Lorsque la révolution islamique a apporté la preuve, qu'en Iran, nous nous trouvions, entre autres, dans une situation où s'opposaient un État faible et une société civile forte (le clergé iranien et ses relais au sein de la bourgeoisie traditionnelle du bazar formaient une société civile forte, capable non seulement de lancer un défi au pouvoir, mais même de le faire tomber) - et le développement de l'islamisme, avec sa forte capacité d'organisation sociale, en marge de celle de l'État, viendra confirmer cet état de fait - des interprétations ad hoc verront le jour, selon lesquelles l'opposition entre société civile et État instable bloque le développement d'une véritable société civile et empêche l'avènement de la démocratie. Ainsi toute une série d'études essaieront d'expliquer que la civilisation islamique se caractérise par un refus catégorique d'accorder une légitimité à l'autorité politique (Crone, 1980). Pipes considère que le postulat d'un idéal est commun à toutes les religions, mais que seul l'islam fixe dans le détail des idéaux politiques dans son code de conduite. Une fois établis de tels idéaux, impossibles à atteindre, les musulmans sont ainsi amenés à considérer tout gouvernement, sous quelque forme que ce soit, comme illégitime (Pipes, $1981: 62$ ).

Il n'est donc pas rare de se trouver confronté à une interprétation qui fait de l'islam le sujet et la source fondamentale de l'Histoire et de l'avenir des Arabes et des musulmans, en prenant comme hypothèse un islam déterministe et omniprésent. Ces analyses perçoivent les sociétés musulmanes comme un tout fermé, terminé une fois pour toutes, comme si elles n'évoluaient pas sans cesse, transformant ainsi leur identité, leurs conceptions, leur culture et leurs institutions en fonction des circonstances. Or, il n'existe pas d'homo islamicus, dissocié, du point de vue anthropologique, du reste de l'humanité (Corm, 1994 : 118), et il n'est ni juste ni légitime d'affirmer que l'expression «démocrate arabe» est une contradiction dans les termes (David Pryce-Jones, 1989 : 406).

Si Abdallah Laraoui a raison de dire que «l'État arabe va être avant tout un corps et des muscles, doté de peu de cerveau ni d'esprit, dépourvu d'une théorie de la liberté, puissant de par son appareil de coercition, mais faible, en réalité, car privé des appuis moraux, idéologiques et éducatifs indispensables» (Laraoui, 1981: 146), cette situation, imparfaite du point de vue de la démocratie,- une fois prises en compte les particularités propres à la région et à sa culture - n'est pas la conséquence d'une «prédestination tribale, du fait des Arabes, ni religieuse, du fait de l'islam», mais s'explique bien plutôt par l'importation, récente qui plus est, du «modèle démocratique», et de son application à des sociétés segmentées et peu structurées, comme c'est souvent le cas dans le Tiers-Monde, qui n'ont eu ni le temps, ni la stabilité nécessaire pour intérioriser la nouvelle forme de leur État national.

En effet, il est exact de dire que la démocratie, conçue comme la possibilité, institutionnalisée et légitime, de contester le pouvoir en place jusqu'à son éventuel remplacement, de façon pacifique, par la voix des électeurs, est un processus qui n'existe pratiquement pas, à la date d'aujourd'hui, dans le monde arabe et musulman (pas plus d'ailleurs qu'en Afrique, en 
Amérique du Sud ou en Asie). Mais les causes de ce déficit sont à rechercher à la fois dans des facteurs sociologiques exogènes, tels que l'ingérence extérieure, la création, suite à la colonisation, d'États artificiels et la manipulation des minorités, ainsi que dans des facteurs endogènes essentiels. A savoir, l'importance de la légitimité historique des «pères de la patrie» et l'immobilisme de ce processus de légitimité historique qui empêche aujourd' hui encore toute une génération d'avoir accès à la «res publica». Autre facteur-clé, l'économie des ces pays, fondée avant tout sur le principe de la rente, principe par définition anti-démocratique, puisqu'il bloque les sources de richesses et obtient ce faisant l'obéissance civile de la majorité, ce qui à son tour entrave l'émergence de forces sociales capables de remettre en cause ce modèle; celuici empêche de plus les gouvernements de développer leurs liens avec la population par le biais d'obligations mutuelles complexes, telles que le paiement d'impôts en échange d'une participation et d'une intégration au système. A cela s'ajoute la politisation de l'armée, dans des États où l'armée a, en général, joué un rôle-clé dans la construction de la nation et le type de gouvernement, ce qui en fait le plus souvent un acteur fondamental sur la scène politique (Égypte, Syrie, Irak, Libye, Soudan, Algérie).

Tout cela ne signifie pas pour autant que la religion n'ait pas d'importance dans les sociétés musulmanes, au contraire. Le problème se situe, une fois de plus, dans la méthodologie suivie pour analyser les différents éléments. Le déterminisme essentialiste, la tendance à ne pas remettre ces éléments dans leur contexte, mais plutôt à les utiliser pour expliquer l'absence de démocratie, par exemple, pervertissent les conclusions fondées sur de telles hypothèses. Les théories «culturalistes» permettent en fait de convertir en une «exception arabo-islamique» ce qui est en fait la situation dans un grand nombre de régions du monde, surtout celles qui ont subi un processus de colonisation (en Asie, en Amérique Latine et en Afrique sub-saharienne).

Quand nous parlons de «visions culturalistes», nous voulons parler de l'imaginaire que les sociétés occidentales se sont forgées par rapport à l'Orient et au monde arabe; cette façon de voir, loin de refléter la réalité, sert à corroborer le stéréotype culturel habituellement accepté en Occident. Celui-ci domine, voire même détermine la présentation et l'analyse des questions ayant trait à cette région du monde et perpétue une vision, fondée sur un antagonisme qui oppose de façon manichéenne l'Islam et l'Occident (alors qu'en réalité il n'existe ni un Orient ni un Occident monolithique). Dans cette représentation ce qui l'emporte c'est l'idée selon laquelle il s'agit d'un conflit culturel entre notre modèle démocratique (laïque) et un autre modèle inacceptable, caractérisé par les règles religieuses dérivées du Coran.

La représentation «culturaliste» développe la défense d'un cosmopolitanisme ethnocentrique où les valeurs de l'Occident, fruits d'une expérience historique spécifique, sont présentées comme le seul paradigme valable pour toute l'humanité, ce qui signifie, au fond, que la diversité culturelle n'est pas perçue comme une pluralité d'options placées sur un pied d'égalité, mais qu'elle se mesure en fonction d'un barème hiérarchisé sur l'échelle opposant modernité et retard (Martín-Muñoz, 1998). Alors qu'en fait, la question de la démocratie comporte une forte dimension culturelle de type identitaire parce qu'il faut que les sociétés acceptent culturellement cette valeur et ne la perçoivent pas comme une expérience faite par l'Autre. C'est ainsi qu'aujourd'hui, et suite à l'expérience vécue au cours de ce siècle, le principe de l'authenticité culturelle est un critère substantiel de crédibilité pour une bonne partie du monde musulman dont la valeur symbolique d'une grande portée ne doit pas être sous-estimée. La démocratie est un processus qui peut être utilisé pour traduire des valeurs culturelles en structures politiques, mais, il faut le dire, le débat occidental sur la démocratisation dans le monde musulman s'est révélé largement incapable d'admettre cet état de fait. 
En revanche, la visibilité des mouvements islamistes et leurs liens avec les processus électoraux et de libéralisation à la fin des années 1980 stimuleront cet imaginaire «culturaliste» par rapport au débat entre islam et démocratie dans un double sens: comme un prolongement logiquenaturel de cet imaginaire anti-islamique et comme instrument utilisé par les groupes dominants et les élites laiques du monde musulman dans leur luttes contre les islamistes et leur recherche d'appuis occidentaux.

Dans le scénario sociopolitique arabe des années 1990, ce que l'on appelle «fondamentalisme» ou «intégrisme islamique» (et par glissement sémantique souvent «terrorisme»), va avoir une fonction d'alibi et de repoussoir qui justifiera l' autoritarisme et la résistance à la démocratisation, et apportera même un précieux instrument aux élites politiques au pouvoir dans leur «stratégies de survie politique autoritaire», tant sur le plan externe (attirant d'énormes soutiens sécuritaires et économiques) que sur le plan interne (par le biais de la «récupération» des anciennes élites sécularisées opposées jusque-là à l' autoritarisme du régime, et de l'usage de la peur de la guerre et des attentats dans la population).

Si la dynamique de libéralisation et de démocratisation qui a suivi la disparition de l'URSS et de l'ordre bipolaire s'est interrompue aussi radicalement au Sud de la Méditerranée, c'est justement parce que l'alternance démocratique s'incarnait dans la tendance islamiste. En conséquence, on observe un retour en arrière autoritaire, accepté de manière explicite - ou implicite - par l'Occident, où le préjugé historique traditionnel vis à vis de l'islam, hérité de malentendus accumulés et d'une relation interprétée depuis des siècles en termes d'affrontement, tourne aujourd'hui de manière presque exclusive autour de l'islamisme. L'incapacité de l'Occident à voir dans l'islamisme réformiste un mouvement d'opposition politique s'appuyant sur une large base sociale, capable à ce titre d'incarner l'alternance face à de vieilles élites de gouvernement incapables de sortir leur pays des crises qui les étouffent, fera de la «peur de l'islamisme « l'alibi qui va justifier le blocage du processus de démocratisation, voire l'autoritarisme croissant du régime allant de pair avec les violations des droits de l'homme, ce qui fera une fois de plus, du monde musulman un cas à part où les citoyens n'ont pas le droit d'élire librement leurs représentants, sous prétexte qu'ils sont «immatures», et qu'»ils ne savent pas choisir».

Par conséquent, le blanc-seing accordé aux gouvernements des pays arabes et musulmans face à leur principale opposition politique va leur donner la possibilité de déformer l'image des islamistes grâce à la confusion sciemment entretenue entre mouvements radicaux et modérés (ou plutôt entre révolutionnaires et réformistes) de sorte que cette stratégie a réussi à occulter la tendance islamiste réformiste majoritaire, en mettant en avant les groupes révolutionnaires (les GIA, la Gamâ'a, al-Jihâd), même s'il existe de fait de grandes divergences entre eux.

En réalité, mis à part quelques louables exceptions, au lieu d'apporter des éclaircissements, le tapage médiatique et l'énorme masse de littérature sur la question de l'islamisme n'ont fait qu'ajouter encore à la confusion, préméditée ou non, quant aux multiples acteurs du mouvement islamiste et à leurs différents rôles sociopolitiques. Toute une littérature «culturaliste» va se consacrer à la diffusion d'une vision monolithique de l'islamisme, considéré comme étant à priori incompatible avec la démocratie et ressenti comme une menace pour l'Occident. Le déterminisme de cette thèse va ignorer, voire occulter, une réalité sociopolitique beaucoup plus complexe et nuancée où il existe un courant islamiste réformiste qui accepte les règles du jeu politique démocratique, lorsque celles-ci réussissent à émerger, comme on l'a vu avec alNahda en Tunisie, le FIS en Algérie ou les Frères musulmans en Égypte, et comme le prouve 
la participation à la vie parlementaire des Frères musulmans en Jordanie, du Hezbollah au Liban, des trois groupes islamistes qui siègent au Parlement du Koweït, et plus récemment de Al-Islâh wa-l-Tadjîd au Maroc. Ces mouvements islamistes condamnent la violence des extrémistes, et apportent la preuve qu'ils sont capables de cohabiter, et même de conclure des alliances avec des partis non-islamistes, comme on l'a vu avec la Plate-forme de Rome, dans le cas de l'Algérie (Martín-Muñoz, 1997) ou avec le Front d'opposition réunissant les islamistes et la gauche en Jordanie et au Koweït (Dabaghy et de Beauvais, 1996 et Aarts, 1993), ou encore avec la prudente «opposition critique» des députés islamistes marocains face au premier gouvernement d'alternance socialiste (Tozy, 1999) ou bien avec la constitution du parti Al-Wasat en Égypte, animé par la nouvelle génération islamiste des Frères musulmans et des personnalités chrétiennes égyptiennes (Martín-Muñoz, 1999).

\section{DES ACTEURS POLITIQUES ISLAMIQUES}

Contrairement à ce que les thèses «culturalistes» voudraient faire croire, il existe une grande variété d'acteurs qui se réclament de l'islam. D'un côté, l'État postcolonial a institutionnalisé un islam officiel, auquel il a concédé la gestion ultra-conservatrice de la société musulmane en échange d'une légitimité exclusive. Les traditionnalistes, uléma très conservateurs, toujours prêts à s'opposer à la moindre innovation, sont les principaux représentants de cet islam institutionnalisé par l'État, qui fait office d'islam officiel (les muftis de la République dans différents pays, les principaux responsables de El-Azhar, les divers Hauts Conseils des uléma ...). Centré sur la défense de la tradition islamique au sein de la société, ce secteur n'a jamais développé d'action politique organisée et n'a pas non plus de projet de gouvernement. C'est au contraire grâce à ses liens implicites avec le pouvoir en place qu'il peut exercer une grande influence et jouir d'une considérable visibilité sociale. Il va éventuellement critiquer telle ou telle mesure de réforme sociale (surtout en ce qui concerne l'éducation, la sécularisation et le Code du Statut Personnel), mais il va toujours soutenir les orientations politiques les plus importantes pour le régime. Ces gardiens de la tradition, rétrogrades, sur le plan culturel, et intolérants, prennent de l'importance au fur et à mesure que les gouvernements ont davantage besoin d'eux pour contrecarrer la perte de légitimité dont ils sont victimes du fait des islamistes. Les traditionalistes vont donc être politiquement correct, en échange de quoi, ils pourront être présents dans la société. Or, c'est précisément cette conception et cette utilisation de la religion qui a empêché le développement de toute réforme morale et intellectuelle islamique.

En ce qui concerne le concept de "fondamentaliste», il est nécessaire d'apporter des nuances sur une série d'éléments. Si l'on fait abstraction du cadre historique dans lequel s'est développé ce terme, celui du protestantisme nord américain, ce concept se fonde sur le retour aux fondements, c'est à dire aux Écritures, comme point de départ de toute tentative de rénovation. Il peut être comparé à une notion qui s'est développée en Islam bien avant que dans le monde chrétien, celle de usûliyya (reconstruire l'ordre social et politique à partir des fondations - usûl - des premiers temps de l'islam, considérés comme véritablement authentiques et purs, car découlant directement de la révélation et non pas de l'exégèse des hommes). Et de plus, l'idéal de la Ville musulmane, décrite en grande partie par les uléma et les docteurs de la loi, a toujours favorisé le développement de la réforme (al-islâhiyya) en terres d'islam. Proclamer la nécessité de retourner aux origines (al-asâla), à la pureté et à l'authenticité des premières sources sacrées en arguant que, depuis les Omeyades, les principes islamiques auraient été mal interprétés, et par conséquent déviés de leur véritable essence, est un phénomène récurrent dans l'histoire de l'Islam: Almohades au XII ${ }^{\mathrm{e}}$ siècle, Wahhabites au XVII ${ }^{\mathrm{e}}$, mahdisme et salafisme au XIX ${ }^{\mathrm{e}}$, Frères musulmans au $\mathrm{XX}^{\mathrm{e}}$. Tous ces mouvements ont été fondamentalistes, dans leur construction 
théorique de la réforme, mais pour certains le retour aux sources consistera à revenir à une lecture «littéraliste» des Écritures (Coran et Sunna), alors que d'autres voudront réinterpréter, avec l'aide de la raison, un nouvel islam qui rejette, non pas la modernisation, mais l'occidentalisation et la mondialisation en tant qu' instruments de domination de certains pays.

Par conséquent s'il est vrai de dire que le processus intellectuel de formation de l'islamisme est de type fondamentaliste, puis qu'il se fonde en effet sur le principe de la réinterprétation et qu'il refuse bon nombre d'éléments hérités de la tradition, tous les mouvements fondamentalistes musulmans n'en sont pas pour autant islamistes, de même que toutes les formes d'organisation islamiste ne s'expriment pas de la même manière, car il s'agit d'un mouvement sociopolitique en pleine évolution où souvent la théorie doit chercher à reprendre à son compte les conséquences de la pratique. C'est d'ailleurs la pratique qui délimite la ligne de partage entre les tendances islamistes radicales d'une part, qui prônent le recours à la violence contre l'État et les autres courants qui, du fait de leur conceptions réformistes, se situent à l'intérieur du système, sans chercher à le faire tomber par la force. Les premiers, clandestins, minoritaires et beaucoup plus éloignés de la société, s'en tiennent de façon rigide à leurs utopie, d'une Ville islamique "pure», sans aucune altération, et demeurent intransigeants vis à vis de tout ce qui s'en éloigne. Les seconds, majoritaires et en contact permanent avec la société et ses aspirations réelles vont évoluer vers des formes de parti politique et limiter leur action à une opposition à l'intérieur du cadre constitutionnel tout en adaptant leur théorie en fonction des circonstances. Par conséquent, les visions ahistoriques qui conçoivent l'islam comme un système global et intemporel qui favorise l'immobilisme, sont de plus en plus marginalisées, alors que, c'est $a$ contrario ce qui s'est passé avec le wahhabisme en Arabie Saoudite. Cela ne signifie pas qu'il n'y ait plus à l'intérieur de ces partis un certain nombre de maîtres-penseurs ou d' «arrièregarde», rétifs à toute innovation, alors qu'en revanche la jeune génération islamiste se caractérise par son caractère novateur en essayant de s'approprier les espaces et les valeurs universelles de la modernité en partant de ses propres référents islamiques.

L'islamisme est donc un néologisme servant à définir un mouvement réformiste musulman qui porte un projet non seulement socioculturel, mais également politique et qui est aujourd'hui amplement associé à la relève à laquelle aspire la nouvelle génération. La nouvelle génération, très importante sur le plan démographique et sociopolitique (plus de 65\% de la population arabe actuelle), se trouve pour la plupart en porte à faux par rapport au système et proche, directement ou indirectement de la tendance islamiste; elle lui apporte de ce fait un grand capital social (Martín-Muñoz, 1999).Contrairement à l'islam traditionaliste et institutionnalisé, les mouvements islamistes sont autonomes sur le plan politique et proches des changements sociaux et politiques qui traversent aujourd'hui les sociétés musulmanes.

Les gouvernements sont aujourd'hui confrontés à ce que l'on pourrait appeler la troisième génération d'islamistes, qui a bénéficié de l'accès à l'éducation d'une bonne partie de la jeunesse et qui est de plus en plus mécontente des systèmes sociopolitiques en vigueur. La mobilisation islamiste connaitra un renouveau et une grande expansion dans les années 1980 , qui s'explique non seulement par l'influence morale exercée par le triomphe de la révolution iranienne, le déclin du modèle socialiste panarabiste ou encore les facilités accordées de façon conjoncturelle dans les années 1970 par certains gouvernements dans leur lutte contre l'opposition de gauche, mais aussi et surtout parce que les islamistes sont perçus comme une nouvelle élite qui représente la contestation de cet ordre immobile sur le plan politique (blocage de la rénovation des élites), économique (entrave à la mobilité sociale et système de corruption 
généralisée qui freine la productivité) et socioculturel («establishment» islamique traditionaliste qui censure la réforme et la rénovation).

Du point de vue de l'action politique, exception faite des groupes violents qui sont minoritaires' et jouissent d'une grande couverture médiatique sans rapport avec leur importance réelle, l'évolution de cette troisième génération islamiste indique clairement qu'elle se situe dans le cadre du territoire de l'État-nation (par opposition aux théories panislamistes) et qu'elle passe par un processus de maturation politique, fondée sur le pragmatisme et les leçons tirées de l'échec de la voie insurrectionnelle. Cette expérience leur a permis de ne pas s'agripper à un conservatisme socioculturel, et les a au contraire poussé sur la voie de la culture du consensus vis à vis d'autres projets sociopolitiques, différents du leur (dans le cadre du pluralisme politique, des élections et du gouvernement).

De fait, cette évolution a favorisé une réflexion approfondie sur ce que signifie être musulman aujourd'hui, pour essayer de mettre en place un ordre islamique qui soit à la fois moderne et authentique, ce qui a permis d'ouvrir un débat très intense sur les relations entre islam, État et démocratie, en relançant l'analyse théorique sur les éléments de l'islam qui connaissent des transformations (al-mutagayyir), c'est-à-dire ceux que la raison humaine peut déduire des textes par un effort d'interprétation structurée, et les éléments ayant trait au gouvernement ou à l'autorité (al-hukm) et aux obligations sociales (mu'amalat). La participation politique et la revendication de la démocratisation trouvent leur explication en termes islamiques, dans la mesure où ces concepts sont compatibles avec celui de Shurâ.

Par exemple, Muhammad 'Imara interprète la Shurâ comme étant «la participation à la gestion des affaires de l'État et de la société « ('Imara, 1993). Fathi Othman en arrive à la conclusion que la Shurâ signifie « une participation active et efficace au processus de prise de décision. Rachid Al-Barawi affirme que l'interprétation démocratique de la Shurâ découle du texte même du Coran et que le régime du parti unique s'oppose en réalité à l'esprit pluraliste islamique (Barawi, 1975: 118). Le but recherché par ces intellectuels consiste à essayer d'adapter et à donner une légitimité, du point de vue de l'islam, aux principes démocratiques de participation et de représentation. C'est dans cette mesure qu'il est important de ne pas sousestimer le rôle de passerelle qu'ils peuvent avoir vis à vis d'une société qui tente de s'approprier de façon endogène, les valeurs de la modernité.

Cependant, et à la grande frustration de ces islamistes libéraux, leurs réflexions et leurs débats sur le pluralisme et la démocratie sont en général ignorés par les «experts» et les médias qui semblent préférer le sensationnalisme du discours extrémiste qui captive les opinions publiques occidentales. De plus, s'il est difficile d'avoir accès à cette pensée politique, c'est aussi parce que les textes sont rédigés pour la plupart en arabe et qu'ils ne trouvent pas d'écho suffisant ni du côté des «experts», ni des maisons d'édition, ce qui les confine à un petit cercle d'initiés.

L'acceptation du multipartisme (al-ta'ddudiyya) et de la répartition du pouvoir, la participation toujours plus active aux institutions de l'État, dans le cadre des réformes libérales mises en œuvre depuis la fin des années 1980, rapprochent les islamistes de la culture démocratique, et ce d'autant plus qu'il existe par ailleurs des politiques gouvernementales autoritaires, visant à les exclure de la scène politique et il est curieux de constater que ce sont justement ces politiques qui reçoivent le soutien et la bienveillance des démocraties occidentales, alors qu'elles donnent souvent lieu à des «bavures» violentes. Rachid Gannouchi, leader du mouvement islamiste tunisien, partisan de l'ancrage des valeurs démocratiques dans la culture islamique, affirmait avec une certaine ironie, lors d'une conférence à l'Université Islamique Averroes de Cordoue, 
en 1995 que le problème aujourd'hui, dans le monde arabe, c'est «de convaincre les laïques de la nécessité de la démocratie, et non pas les islamistes».

Gannouchi, dans les multiples interviews et déclarations réalisées de son exil en Europe, s'est déclaré «contre le fondamentalisme qui croit posséder à lui seul la vérité et veut l'imposer aux autres» et sur la question de l'islam et la démocratie, il pense que «l'islam ne contient pas de programme précis quant à notre vie (...), il exprime plutôt des principes généraux, notre tâche consistant à formuler ce programme grâce à l'interaction entre les principes islamiques et la modernité». Les croyants ont pour garantie l'ijtihad, qui leur permet d'interpréter le texte coranique, et leur capacité d'action est totale en la matière étant donné que l'islam n'a aucune institution, ni d'autorité unique qui puissent représenter la foi ou contredire les interprétations (Wright, $1996: 73$ ).

En outre, certains textes islamistes parmi les plus récents considèrent que la participation politique est nécessaire aujourd'hui pour relever les défis que leur pose le monde moderne, et d'autres vont même jusqu'à concevoir la participation comme un antidote à la violence (alNafisi, 1989). Cela dit, il est vrai qu'il existe, au sein de l'islamisme réformiste, des points de vue qui posent des objections à une telle participation, comme Jamal Al-Banna (1986) ou le Jordanien Abd al-Aziz Al-Khayyat (1992). Le maintien au pouvoir de gouvernements autocratiques et le manque de démocratie sont vus par les islamistes comme l'une des causes principales de violence, et d'ailleurs, les expériences auxquelles ils participent et où ils occupent des sièges au Parlement (au Yémen, en Jordanie, au Liban et en Malaisie) sont présentées comme des exemples où la démocratisation a permis d'arrêter la violence (al-Wa'i, 1993 : 39). De plus, ils dénoncent la politique de double langage des démocraties occidentales qui ne sont guère exigeantes en matière de démocratie et de respect des droits de l'homme vis à vis des pouvoirs en place, alors qu'elles adressent ces reproches exclusivement aux islamistes. On a même vu les pays occidentaux défendre le non-respect du résultat des élections démocratiques, lorsque celles-ci donnaient la victoire aux islamistes.

De même, l'évolution politique en Iran a apporté la preuve que la «raison d'État» l'emporte peu à peu sur la «logique révolutionnaire» et qu'un point de vue réformiste et ouvert a fait pas à pas son chemin face au discours moraliste et intolérant. D'ailleurs, aujourd'hui, des journalistes, des universitaires et des militants politiques discutent et même reposent la question du rôle de l'islam en politique et de la participation du clergé au gouvernement (peut-il y avoir une interprétation définitive de l'islam ? quel est le rôle de la religion en politique ? l'islam est-il compatible avec la démocratie ? Est-ce-que l'expérience postrévolutionnaire de l'Iran entraîne la nécessité de réformer l'ordre religieux traditionnel ?). Tout ceci ouvre le débat sur les mérites du pluralisme, de la participation politique et des rapports avec le monde extérieur, y compris, bien entendu avec l'Occident.

L'un des penseurs les plus éminents de ce courant politique réformiste musulman, le professeur Abdolkarim Soroush ${ }^{2}$ représente précisément ce vaste mouvement islamique qui réclame le pluralisme et la participation politique, en les considérant comme intrinsèquement islamiques.

Ils apportent ainsi la preuve qu'il existe un substrat commun permettant une interaction fructueuse entre les sociétés musulmanes et occidentales, et battent en brèche l'idée habituellement répandue selon laquelle la société iranienne est monolithique et à jamais hostile à l'Occident. Soroush est un penseur controversé qui défend l'idée que les valeurs démocratiques et les droits de l'homme ont leur place dans tout gouvernement islamique, et qu'il ne peut y avoir de vision définitive et totale de l'islam (rejetant donc toute tentative visant à formuler une 
idéologie politique islamique officielle).Il propose des réformes fondamentales de l'ordre religieux traditionnel (que les analystes occidentaux appellent généralement «le clergé shiite») et il ne rejette pas le rôle de l'islam dans la politique en général. Il affirme, d'ailleurs, qu'un gouvernement démocratique doit refléter la société qu'il représente. Si l'Iran est un pays musulman, son gouvernement doit avoir un caractère islamique, dit Soroush, mais les droits de l'homme doivent servir de référence au gouvernement de manière à garantir la nature démocratique de l'État religieux (Vakili, 1996).

Ce qui est particulièrement significatif dans ce raisonnement, c'est qu'il n'est pas le fait d'un intellectuel iranien laïcisé, mais d'un musulman fervent qui a participé aux premiers moments du gouvernement postrévolutionnaire, et qui formule entièrement sa pensée politique à partir de sa conception de l'islam. La compatibilité entre sa vision de l'islam et ses idées politiques donne une grande valeur pratique à cette pensée critique. Soroush pose ainsi d'un autre point de vue une question souvent posée par d'autres intellectuels musulmans sur la nécessité de «reconstruire» l'islam pour l'adapter aux besoins de l'homme moderne. Sa réponse consiste à dire qu'il ne s'agit pas de reconstruire l'islam, mais plutôt la compréhension que l'homme a de l'islam. Une fois établie cette distinction, Soroush est en mesure de réconcilier une religion qui ne bouge pas et un monde en mouvement. C'est à dire qu'il ne faut pas confondre la religion elle-même et la connaissance religieuse qu'on peut en acquérir par l'étude. Si l'on accepte qu'une telle confusion constitue une erreur, on comprend par la même occasion que la connaissance religieuse est une construction humaine, et donc à fortiori en continuelle évolution. Les musulmans doivent donc «reconstruire» leurs interprétations religieuses en accord avec leur compréhension mouvante de leur propre monde. Soroush pense également que toute forme d'idéologie islamique est un obstacle au développement de la connaissance religieuse. Cependant, même si Soroush rejette le rôle de l'islam utilisé en tant qu'idéologie, que ce soit comme instrument révolutionnaire ou comme plate-forme de gouvernement, car cela implique une vision statique et officielle de l'islam qui affaiblit la légitimité du pouvoir, il n'en appelle pas pour autant à la séparation entre religion et politique. Il argumente au contraire que dans une société religieuse la politique acquiert forcément une dimension religieuse. Pour Soroush, la question qui se pose n'est pas celle de la séparation, mais de la nature de l'interaction entre les deux. Il rejoint ainsi la thèse de Ali Shariati sur la «géographie des concepts», selon laquelle les concepts doivent être appliqués dans leur lieu d'origine et pas nécessairement dans d'autres contextes culturels. En d'autres termes, si la laïcité a permis à l'Occident de se libérer de l'oppression religieuse, les sociétés musulmanes ont une autre expérience historique qui leur est propre.

\section{LES ISLAMISTES ET L'OCCIDENT}

L'intellectuel islamiste Youssouf al-Qardawi, qui jouit d'une certaine influence, se demandait pourquoi les Européens acceptent qu'il existe des partis chrétiens, dans leur dénomination et leur esprit et qu'ils refusent ce droit aux musulmans (Qardawi, 1993 : 30). Ces contradictions sont ressenties comme une discrimination à leur égard, auxquelles s'ajoutent les conséquences de la politique américaine, fondée sur un soutien aveugle apporté à Israël alors que celui-ci n'a respecté aucune des résolutions des Nations unies sur les droits des Palestiniens et que, dans le même temps, les États-Unis sont d'une intransigeance radicale lorsqu'il s'agit des voisins arabo-musulmans d'Israël (bombardements contre la Libye, le Soudan et l'Irak). Tout ceci pousse les islamistes à accentuer leur différence et leur distance vis-à-vis de l'Occident. 
La perception dominante que l'Occident a des islamistes, et c'est bien là le problème, ne lui permet pas de voir qu'il existe différents islamismes et que selon leur environnement national, leur terrain social ou leurs modes d'action, ils peuvent varier considérablement. Il continue de prévaloir une vision monolithique, qui choisit soit le discours le plus traditionaliste-qui a priori se veut intemporel et imperméable à l'histoire - ou bien le plus sensationnaliste - venant des secteurs les plus insatisfaits, illettrés ou radicalisés - suite à quoi, à partir de ces deux groupes situés aux extrêmes du spectre, on en arrive à des conclusions, largement déformées du fait de la méthodologie suivie, sur la nature globale de l'islamisme. Avec de tels critères de sélection, on oublie que les différentes manières dont le politique s'approprie le religieux dépendent bien moins de l'origine des acteurs, en terme de dogme, que de la réalité sociologique qui les caractérise. Dans le cas des islamistes, ils se retrouvent en majorité dans des tendances réformistes qui se situent dans l'énorme espace du centre, la plupart de temps occulté, entre les traditionalistes et les groupes violents.

Par conséquent, l'interprétation des troubles politiques sur la rive sud de la Méditerranée est en grande partie influencée par les représentations négatives en vigueur quant au «fait islamique». Ces représentations se nourrissent, pour l'essentiel, de la longue accumulation de malentendus historiques, où l'Islam en contact permanent avec l'Europe pour des raisons géographiques, humaines et commerciales, a joué le rôle de repoussoir culturel, dans l'alchimie complexe du processus de construction de l'identité.

C'est à cause de cet imaginaire dominant que les islamistes réformistes ont tant de mal à communiquer avec l'Occident et à faire comprendre qu'ils ne sont pas les mouvements violents et radicaux qu'on décrit. Ce manque de communication vient également de l'absence de passerelles intellectuelles communes, comme cela avait été le cas avec les mouvements tiersmondistes ou nationalistes qui avaient des liens idéologiques avec la gauche européenne. Cela s'explique en grande partie par la tendance démesurée de l'Occident à «occidentaliser» l'histoire universelle, ce qui l'amène à rechercher son propre système de valeurs chez ses interlocuteurs. Les acteurs qui n'entrent pas dans ses références idéologiques et symboliques ne pénètrent pas facilement dans les circuits de communication (Martín-Muñoz, 1999).

Il nous est, à partir de là, difficile d'accepter, par exemple, qu'il n'y ait pas eu dans l'expérience historique et sociologique du monde arabe de conflit radical entre la Raison et la Foi, qui a caractérisé la construction moderne de l'Europe. Or, si celle-ci s'est développée sur la base d'une conception linéaire de la modernité, où la perte de vitesse du référent religieux a été de pair avec les progrès de la modernité, et cela n'a pas été le cas dans le monde musulman.

Alors qu'en Europe la laïcité s'est affirmée comme une valeur de modernité et de démocratie, dans les pays arabes, la laïcité n'a jamais été que le fruit du volontarisme autoritaire des leaders nationalistes, de la période postcoloniale. Car, en effet, ces sociétés n'ont jamais connu de vaste processus social de sécularisation. Et de plus, dans la mesure où la laiicité, dans les pays musulmans, a été le plus souvent favorisé par des élites dirigeantes autoritaires, assises sur le patrimoine du pays, il existe, contrairement à l'Europe, un conflit potentiel d'intérêt entre démocratie et laïcité. En outre, comme la laïcité a souvent été imposée par opposition à l'héritage islamique, il existe également un conflit potentiel d'intérêt entre laïcité et identité culturelle.

A quoi peuvent bien nous servir alors ces versions déterministes qui font de l'islam un obstacle à la démocratie, alors que ce sont justement les gouvernements et les élites qui s'autoproclament modernes et laïques qui entravent le développement de la démocratie dans les sociétés musulmanes? 


\section{BIBLIOGRAPHIE}

AARTS, P. 1993. «Le Koweït d'après-guerre et le processus de démocratisation». MaghrebMachrek, 142. 61-79.

BADIE, B. 1986. Les Deux États. Paris, Fayard.

Al-BANNA, J. 1986. Al-Hukm bi-l-Qur'ân wa qadiyyat tatbîq al-hukm. Al-Qâhira.

Al-BARAWI, R. 1975. Al-Qur'ân wa-l-Nuzûm al-Ijtimâ'iyya al-mu 'âsira, Le Caire.

Al-BICHRI, T. 1991. «Les conditions d'un dialogue avec l'Occident». Egypte/Monde Arabe, 7. 125-139.

BILL, J. A. \& SPRINGBORG, R. 1984. Politics in the Middle East. New York, Harper Collins.

CORM, G. 1994. «Perspectives démocratiques au Machrek». In: R. Bocco \& M.R. Djalili (eds), Moyen-Orient: migrations, démocratisations, médiatisations. Paris, PUF. 118-119.

CRONE, P. 1980. Slaves on Horses: The Evolution of the Islamic Polity. Cambridge. Cambridge University Press.

DABAGHY, J. DES BEAUVAIS, C. 1996. «Hussein, le funambule de la paix». Arabies, 111, Mars. 12-21.

HUNTINGTON, S. 1984. «Will more countries become democratic ?». Political Science Quarterly 99, 2. 193-219.

'IMARA, M. 1993. «Al-ta 'addudiyya: al-ru'ya al-islâmiyya wa-l-tahaddiyât al-garbiyya». In: Nadwat al-Ta'ddudiyya al-Hizbiyya wa-l-Ta'ifiyya wa-l-'Irqiyya fî-l- 'Âlam al-'Arabî. Herndon, VA: IIIT. 3-14.

Al-KHAYYAT, 'A. Al-'A. 1992. «Al-Ta'addudiyya min wiyhat nazar islâmiyya». AlLiwa',16, 1, Octobre, 21.

LAROUI, A. 1981. Mafhûm al-Dawla. Casablanca, Al-Markaz al-Thaqâfî al-'Arabî.

LEWIS, B. 1996. «Islam and Liberal Democracy. A historical Overview». Journal of Democracy, April, pp. 52-63.

MARTIN-MUÑOZ, G. 1997. «Le régime algérien face aux islamistes». In: B. Kodmani \& M. Chartouni (eds) Les États Arabes face à la contestation islamiste. Paris, Armand Colin/ IFRI. 41-70.

1998. «The image of Islam and the Arabs in the West. The prevalence of culturalist visions». In: J. Nielsen \& S. A. Khasawnih, (eds.) The Arabs and the West: Mutual Images. Amman, Jordan University Press. 59-72.

1999. Islam, Modernism and the West. London \& New York, IB Tauris.

1999. El Estado Arabe. Crisis de legitimidad y contestación islamista. Barcelona, Bellaterra.

Al-NAFISI, 'A. 1989. Al-Haraka al-islâmiyya. Ru'ya mustaqbaliyya: Awrâq fî l-Naqd alDhatî. Al-Qâhira, Maktaba Madbuli.

OTHMAN, F. 1992. Fî̀-l-Tajrîba al-siyâsiyya li-l-haraka al-islâmiyya al-mu 'âsira (n.é). 
PIPES, D. 1981. Slave, Soldiers and Islam: The Genesis of a Military System. New Haven: Yale University Press.

PIPES, D. 1983, In the Path of God: Islam and Political Power. New York, Basic Books.

PRYCE-JONES, D. 1989. The Closed Circle: an interpretation of the Arabs. Nueva York, Harper \& Roww.

Al-QARDAWI, Y. 1993. Al-Mujtama', 24, 1081. 30-36.

SADOWSKY, Y. 1993. "The new orientalism and democracy debate». Middle East Report, $183,23,4,1993.14-21$.

TOZY, M. 1999. «Réformes politiques et transition démocratique». Maghreb-Machrek, $164.67-84$

VAKILI, V. 1996. Debating religion and politics in Iran: The political thought of Abdolkarim Soroush. Studies Department Occasional Papers Series, 2. Council on Foreign Relations, NY.

Al-WA'I, T. 1993. «Al-hiwâr huwa al-hall li-1‘Unf». Al-Mujtama‘, 24, 1081, Décembre, 28. 38-41.

WRIGHT, R. 1996. «Two visions of reformation». Journal of Democracy, April. 64-75.

\section{NOTES}

I L'expérience de la répression sous le régime de Nasser fera naître un courant de pensée radical chez les Frères Musulmans, représenté par Sayyed Qutb. Les idées de Qutb sur le takfir actif, qui consiste à excommunier la société dans son ensemble, et ferment d'une jihâd conçue comme une action révolutionnaire intérieure, va servir d'unspiraion aux dissidents extrémistes de Tanzîm al-Jihâd, Hizb al Tahrîr, Takfir wa Hijira et plus récemment au GIA algérien et à la Gamâ 'a islâmiyya de Haute-Égypte.

2 Abdolkarim Soroush est le pseudonyme de Hossein Dabbagh. Il a étudié au lycée Alavi, un des premiers lycées dont l'enseignement comportait à la fois des sciences exactes et des études islamiques, et par lequel sont passés bon nombre de membres du gouvernement actuel. Il a fait ses études de pharmacologie à Téhéran, et celles d'histoire et de philosophie en Angleterre. Il a été très proche d'Ali Shariati (décédé en 1977) et de l'ayatollah Murtaza Mutahhari (décédé en 1979). Il a occupé un poste élévé au Comité Culturel de la Révolution jusqu'en 1987, date à laquelle il donna sa démission pour cause de divergences sur la pratique suivie.Il a créé en 1992 la Faculté de Recherches Historiques et de Philosophie de la Science au sein de l'Institut de Recherche en Sciences Humaines de Téhéran. Soroush dispose d'une grande audience auprès des jeunes Iraniens, des intellectuels et des hommes politiques réformistes, mais il compte également de nombreux adversaires au sein du secteur le plus conservateur du régime. Au fur et à mesure que ses conférences à l'Université et ses interventions dans les mosquées attiraient de plus en plus de monde, les conservateurs essayèrent de les boycotter, et Soroush finit par les supprimer et par se consacrer en 1996 à voyager et à donner des cours à l'étranger, parce qu'il craignait pour sa vie, comme il l'a expliqué dans une lettre ouverte au président Rafsanjani. Il est par ailleurs très significatif que le guide spirituel du pays, Ali Khamenei, ait consacré plus de temps, dans son discours prononcé à l'occasion de l'anniversaire de la prise de l'ambassade américaine, à critiquer les idées de Soroush que les États-Unis. 